\title{
Neue Angebote entwickeln und vermarkten: zwei Beispiele
}

\author{
"Tandem - Reisen mit Betreuung«: Gemeinsam die \\ Welt entdecken
}

Seit Januar 2000 gibt es bei der Lebenshilfe Worms mit »Tandem - Reisen mit Betreuung « ein Reisebüro für Menschen mit Behinderungen.

»Tandem-Reisen « bietet betreute Urlaubsreisen für Menschen mit einer geistigen oder mehrfachen Behinderung an. Die Reiseteilnehmerinnen und Reiseteilnehmer werden während des gesamten Urlaubsaufenthaltes individuell je nach Art und Schwere ihrer Behinderung von geschulten Mitarbeiterinnen und Mitarbeitern unterstützt und gepflegt. Unsere Reisebegleiter sind 24 Stunden täglich vor Ort und stehen durchgängig als Ansprechpartner zur Verfügung. Die Freizeitaktivitäten am Urlaubsort werden auf die Wünsche und Bedürfnisse der Teilnehmer abgestimmt, sodass jeder - unabhängig von Art und Schwere der Behinderung - daran teilnehmen kann.

Die Reiseleitungen und Reisebegleitungen werden von ehrenamtlichen Mitarbeiterinnen und Mitarbeitern übernommen, die diese Arbeit zusätzlich zu ihrem Hauptberuf oder ihrer Ausbildung (Studium oder Lehre) machen. Tandem-Reisen bietet Schulungen an, an welchen jeder Mitarbeiter teilnehmen muss, um sich unabhängig von seiner eigentlichen Profession, pädagogisch, pflegerisch und rechtlich auf seine Aufgaben vorzubereiten.

»Tandem-Reisen mit Betreuung « hat es sich zum Ziel gesetzt, Menschen mit geistiger oder mehrfacher Behinderung die Möglichkeit zu bieten, Urlaub zu machen wie jeder andere auch. Den Menschen mit Behinderung wollen unabhängig von Angehörigen oder Wohnheimmitarbeitern auf Reisen gehen und neue Leute kennenlernen. Nicht selten sind in den vergangenen Jahren neue Freundschaften entstanden, die auch über größere Entfernung Bestand hatten und zu gemeinsam geplanten Reisen im folgenden Jahr führten.

Unter der Leitung einer Sozialpädagogin geht es »Tandem-Reisen « nicht um den optimalen Urlaub im Sinne des klassischen Reiseverkehrs, sondern vielmehr um den optimalen Urlaub für Menschen mit Behinderung: rollstuhlgerechte und behindertenfreundlichen Unterkünfte, individuelle Betreuung und Pflege, lebenspraktische Unterstützung, bedürfnisgerechte Freizeitaktivitäten.

Die Idee $\mathrm{zu} »$ Tandem-Reisen « entwickelte sich aus den Freizeitaktivitäten des Familienentlastenden Dienstes (Offene Hilfen) der Lebenshilfe Worms. Dessen zwei oder

\section{Katja Münzing}

Katja Münzing (33) ist Diplom-Sozialpädagogin mit der Zusatzqualifikation Touristikfachkraft. Bei der Lebenshilfe Worms hat sie ab dem Jahre 2000 die »Tandem-Reisen « aufgebaut.

Tandem-Reisen mit Betreuung, Lebenshilfe Betreuungs- und Beförderungs-GmbH, Kurfürstenstraße 1-3, 67549 Worms, Telefon 06241 203-8130, Fax 06241 203-8111, E-Mail info@lebenshilfeworms.de, Internet http://www.tandem-reisen.de

drei »Freizeitmaßnahmen « im Jahre konnten den Bedarf und die hohe Nachfrage der Interessenten nicht mehr abdecken. Die Gründung von »Tandem-Reisen « ermöglichte nun in den vergangenen Jahren den Auf- und Ausbau eines umfangreichen Reiseprogramms: Im Jahr 2006 kamen zu den 55 geplanten und im Reiseprogramm vorgestellten Reisen, noch 23 Kooperations- und Individualreisen hinzu. Als Kooperationsreisen werden die Fahrten bezeichnet, die in Zusammenarbeit mit anderen Einrichtungen der Behindertenhilfe stattfinden: Es melden sich neben den privaten Kunden vermehrt Werkstätten und Wohneinrichtungen für Menschen mit Behinderung, die einen Teil ihrer Freizeitmaßnahmen extern von »Tandem-Reisen « organisieren und durchführen lassen. Die bestehenden Gruppen aus den Institutionen werden meist von einrichtungsfremden Teilnehmenden ergänzt, sodass einmal mehr die Möglichkeit zum Kennenlernen neuer Menschen gegeben ist.

»Tandem-Reisen« bietet auch die Möglichkeit an, individuelle Urlaubsreisen zu machen. In diesem Fall können Einzelpersonen Ziel, Dauer Zeitpunkt und Unterkunftsform angeben, anhand dessen ein kompletter Urlaub organisiert wird. Der Reisende wird für die Reisezeit einen Mitarbeiter an der Seite haben, der ihn unterstützt und begleitet.

Urlaubsziele von Tandem-Reisen waren und sind: Mallorca, Toskana, Gran Canaria, Türkei, Tunesien, Dänemark, Österreich, USA, Ägypten, Griechenland. Auch viele Orte und Regionen in Deutschland sind beliebte Urlaubsziele: Berlin, Mecklenburgische Seenplatte, Bodensee, Allgäu, Schwarzwald, die Nord- und Ostsee. Auch Event-Reisen zu Musicals, Sportveranstaltungen (Formel 1, Fußballspiele etc.) oder Kreuzfahrten zählen zu den Angeboten. Einige dieser Angebote sind Reisen, die speziell für Senioren, Junio- 
ren, Kinder und Jugendliche oder Menschen mit hohem Betreuungsbedarf ausgeschrieben werden.

Reisezeit ist bei »Tandem-Reisen « das ganze Jahr: Wir beginnen im Januar mit einer Winterreise, nutzen die Frühlings- und Sommermonate für 5- bis 14-tägige Erholungs- und Eventreisen und schließen das Jahr mit der traditionellen Adventreise und einer Silvesterreise ab. Die Reiseangebote werden nicht nur von Menschen aus Worms und der Umgebung genutzt, sondern von Reisefreudigen aus ganz Deutschland.

Die Devise von »Tandem-Reisen mit Betreuung « ist: Urlaubsreisen sind ein selbstverständlicher Bestandteil des Lebens von Menschen mit einer geistigen oder mehrfachen Behinderung.

\section{Die andere Kontakt- und Partneragentur: "Schatzkiste« für das kleine Glück}

Das Sozialpsychiatrische Zentrum Haus am Landwehrweg in Mainz betreibt seit Mai 2006 die erste Kontaktund Partnervermittlung für Menschen mit Behinderungen in Rheinland-Pfalz.

Die »Schatzkiste« soll Anlaufstelle sein für alle Menschen mit Behinderung oder psychischen Problemen in Mainz und Umgebung, die den ernsthaften Wunsch nach neuen Kontakten oder einer Partnerschaft haben. Die Diplom-Sozialpädagogin und Ansprechpartnerin der "Schatzkiste« Eva Mais: »Die Freizeit- und Partnerwünsche von Menschen mit Behinderung unterscheiden sich kaum von denen anderer Leute. Allerdings sind ihre Chancen zur Erfüllung geringer. Die `Schatzkiste< will ihnen helfen, Menschen mit ähnlichen Interessen und Hobbys kennen zu lernen - spätere Liebe nicht ausgeschlossen. Die Vermittlung ist übrigens kostenlos. «Vorbild der Mainzer »Schatzkiste « ist eine gleichnamige, seit 1988 sehr erfolgreich tätige Hamburger Einrichtung.

Das Angebot der Kontakt- und Partnervermittlung ist ein Beispiel für die gelungene Umsetzung eines Zentrumsgedankens: Kerngeschäft in unserem Haus sind stationäre und ambulante Angebote der Teilhabeplanung und Begleitung. Erst durch offene Angebote, wie die »Schatzkiste« und öffentliche Veranstaltungen innerhalb der Gemeinde oder des Stadtteils, wird das Haus zur Begegnungsstätte für Behinderte und Nichtbehinderte. Das Sozialpsychiatrische Zentrum wird auf diesem Weg mit Leben erfüllt und dient nicht nur eine Summe von Dienstleistungsaufträgen, sondern ist gekoppelt an Normalitäts- und bürgerschaftliche Prozesse. Wirksam auch im Sinne einer Öffentlichkeitsarbeit für unsere üblichen Angebote, die unser ökonomisches Überleben sichern.

Die Idee der »Schatzkiste« wurde im Übrigen auch aufgegriffen, weil wir in unseren Hilfeplanungsgesprächen immer wieder von den Menschen gehört haben: »Ich hätte so gerne eine Partnerin oder einen Partner.« Menschen mit Behinderung bleiben die üblichen Wege der Kontaktanbahnung oder Partnersuche meist verschlossen. Die Freizeitaktivitäten sind reduziert, Kontaktanzeigen in Medien oder gar die Einschaltung teurer Partneragenturen sind meist weniger mit Erfolg als mit Frust verbunden.
Das soziale Netz von Menschen mit Behinderung ist meist sehr weitmaschig, sofern überhaupt vorhanden. Ihre Kontaktmöglichkeiten beschränken sich weitgehend auf Schulen, Tages- oder Werkstätten, Kliniken und andere soziale Einrichtungen. Für viele Behinderte bleiben große Lebensträume unerfüllbar, aber die Chancen für das kleine Glück stehen durchaus gut - was wir mit der »Schatzkiste« beweisen wollen.

Am Beginn der individuellen Suche steht die persönliche Anmeldung der Interessenten im Haus am Landwehrweg zu den »Schatzkiste«-Öffnungszeiten oder nach Terminabsprache. Bei diesem ersten, etwa einstündigen Gespräch werden die Interessenten ausführlich nach ihren Vorlieben, Wünschen und Hobbys befragt. Die Angaben werden in einer Computerdatei gespeichert und durch ein vor Ort gemachtes Foto ergänzt. Eva Mais vergleicht später die Angaben mit denen der anderen Kandidaten in der Kartei und schlägt bei hinreichender Übereinstimmung einen passenden Partner vor. Der Interessent erhält zunächst nur ein Foto per Post. Die Bekanntgabe weiterer Daten hängt von der Entwicklung ab und davon, ob der Vorgeschlagene der Weitergabe ausdrücklich zugestimmt hat. Die »Schatzkiste« hilft bei der Kontaktaufnahme und lädt auf Wunsch die möglichen Partner und eventuelle Begleiter ins Büro ein. Natürlich können sich die Partner auch selbstständig verabreden und treffen.

Seit Mai 2006 wurden rund 70 Personen, meist mit psychischen Beeinträchtigungen, in die »Schatzkiste«-Kartei aufgenommen und mehrere Kontaktanbahnungen gestartet. Obwohl die Mainzer »Schatzkiste« ausdrücklich auch die Vermittlung von Freizeitkontakten anbietet, stehen überwiegend Partnersuchende in der Kartei. Bisher haben sich überwiegend Männer gemeldet.

Die Schatzkiste soll einen Beitrag dazu leisten, Menschen mit Behinderungen die Teilhabe am gesellschaftlichen Leben zu ermöglichen, ihre Lebensqualität zu verbessern und chronisch erkrankte Menschen stärker in die Gesellschaft eingliedern. Unsere Aufgabe ist es, Menschen mit großer Sensibilität zu unterstützen und ähnliche Angebote vermehrt in unsere Zentren einzubinden. Somit wird die Hemmschwelle der eventuellen Nutzung von professionellen Diensten herabgesetzt und das genaue Hinhören der Fachkräfte bei Bedarfsabfragen zwingend notwendig um neue Angebote mit den betroffenen Menschen gemeinsam zu entwickeln.

\section{Kerstin Diefenbach}

Kerstin Diefenbach (43) ist Diplom-Sozialpädagogin und seit Januar 2007 Gesamtleiterin der Einrichtungen und sozialen Dienste der Gemeinnützigen Gesellschaft für PARITÄTISCHE Sozialarbeit $m b H$ in Mainz.

"Schatzkiste", Sozialpsychiatrisches Zentrum Haus am Landwehrweg, Gemeinnützige Gesellschaft für Paritätische Sozialarbeit $m b H$, Landwehrweg 5, 55131 Mainz, Telefon 06131 55790, Fax 06131 557935,E-Mail leitung@hal-mainz.de, Internet http://www.hal-mainz.de 